\title{
Measuring the Strain Sensitivity in Si (001) Electron Channeling Patterns Using Higher-order Laue Zone Line Shifts
}

\author{
Joel Lammatao ${ }^{1}$, Lisa Chan ${ }^{2}$, Tony Owens ${ }^{2}$, Marc De Graef ${ }^{1}$, and Yoosuf N. Picard ${ }^{1}$ \\ ${ }^{1}$ Carnegie Mellon University, Department of Materials Science and Engineering, Pittsburgh, PA 15213, \\ United States \\ ${ }^{2}$ TESCAN USA Inc., 508 Thomson Park Drive, Cranberry Township, PA 16066, United States
}

Electron channeling is a scanning electron microscopy (SEM)-based diffraction technique in which pattern contrast is generated by the orientation dependence of the backscattered electron yield. Electron channeling patterns (ECPs) not only provide crystallographic information about the crystal (such as phase or orientation), they can also give information about the local strain state. This is possible since positions of higher-order Laue zone (HOLZ) lines, which appear in the ECPs, are very sensitive to the local lattice parameters of the material.

HOLZ lines are utilized to quantify strain by analysis of convergent beam electron diffraction (CBED) patterns obtainable in a transmission electron microscope (TEM) [1]. Their positions may be tracked with adequate precision using Hough transforms [2]. However, to our knowledge, this has not been extended to SEM-based electron channeling, which has its advantages over TEM in terms of the relative ease of sample preparation and applicability over large areas $\left(>5-10 \mu \mathrm{m}^{2}\right)$. In order to assess its merits for measuring strain, it is necessary to quantify the strain sensitivity for this technique.

Twenty-one ECPs were acquired from a $\mathrm{Si}(001)$ single-crystal substrate over a range of accelerating voltages from 19.90 to $20.10 \mathrm{kV}$ in $0.01 \mathrm{kV}$ increments. This was accomplished using two SEMs: an FEI Quanta 600 field emission gun (FEG)-SEM and a TESCAN Mira-3 FEG-SEM. ECPs were recorded in the FEI SEM by low-magnification imaging over a scan angular range of $4.8^{\circ}$ (Figure 1a-c). Selected area channeling patterns (SACPs) were recorded in the TESCAN SEM in rocking mode over the same angular range (Figure 1d-f). Stage rotation and tilt were carefully chosen to align the (001) zone axis with the optic axis. Small changes in voltage $(0.01 \mathrm{kV})$ were used as a proxy for the effects of hydrostatic strain on the crystal. The strain sensitivity for this study was defined by the smallest detectable change in HOLZ line position that is greater than the measurement uncertainty (determined from eight SACPs recorded at the same accelerating voltage).

For each SACP, a 244 x 244 pixel size region of interest (ROI) was selected (Figure 2). Each ROI was subsequently image-processed (using ImageJ) with the following sequence: (1) a subtraction to reduce background intensity, (2) unsharp masking to enhance contrast near the HOLZ lines, and (3) median filtering to reduce noise (Figure 3(b,e)). HOLZ lines were extracted by implementing a Hough transform routine. The distance between a selected pair of HOLZ line intersections, d, (Figure 3(c, f)) was measured for each ROI as a function of simulated hydrostatic strain (Figure 4). Based on these measurements, a preliminary strain sensitivity of $\sim 0.15 \%$ has been determined. To corroborate this result, the entire procedure has been replicated for a series of dynamically simulated SACPs over the same voltage range, which is in good agreement with the experimental SACPs. This sensitivity measurement improves upon previous attempts for ECP HOLZ line analysis [3]. 
References:

[1] Akaogi, T. et al, J. Electron Microsc. 53 (2004), 593-600.

[2] Kramer, S. and Mayer, J., J. Microsc. 194 (1999), 2-11.

[3] Kozubowski, J.A., Keller, R.R., and Gerberich, W.W., J. Appl. Cryst. 24 (1991), 102-107.

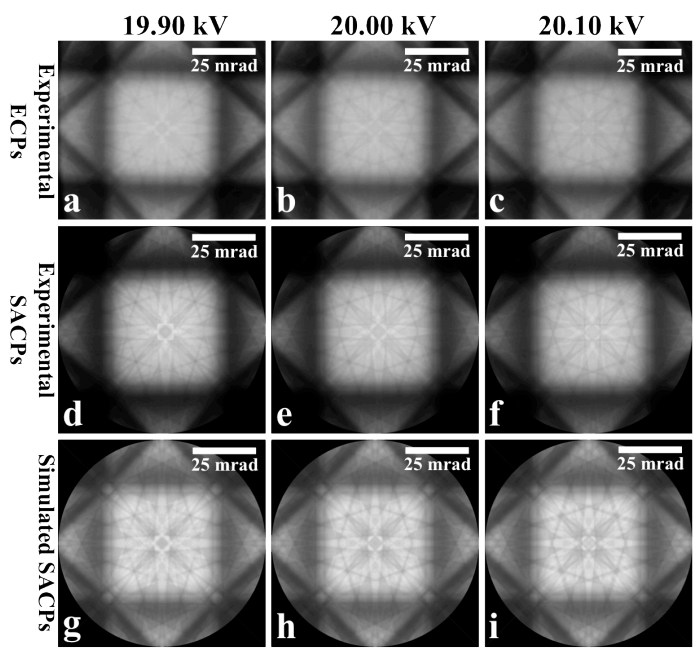

Figure 1. Experimental (a-f) and dynamically simulated (g-i) Si (001) ECPs recorded at varying SEM acceleration voltages.

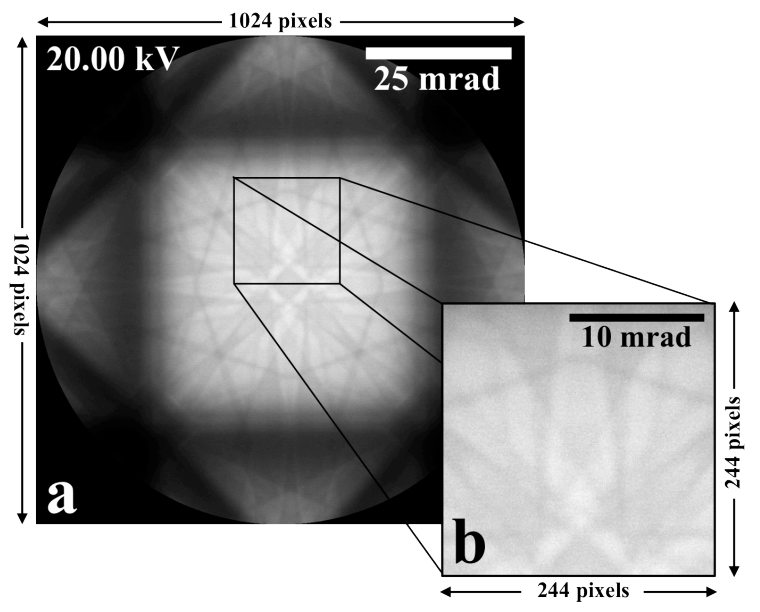

Figure 2. For each experimental $\mathrm{Si}$ (001) SACP (a), a smaller square ROI has been selected $(b)$.
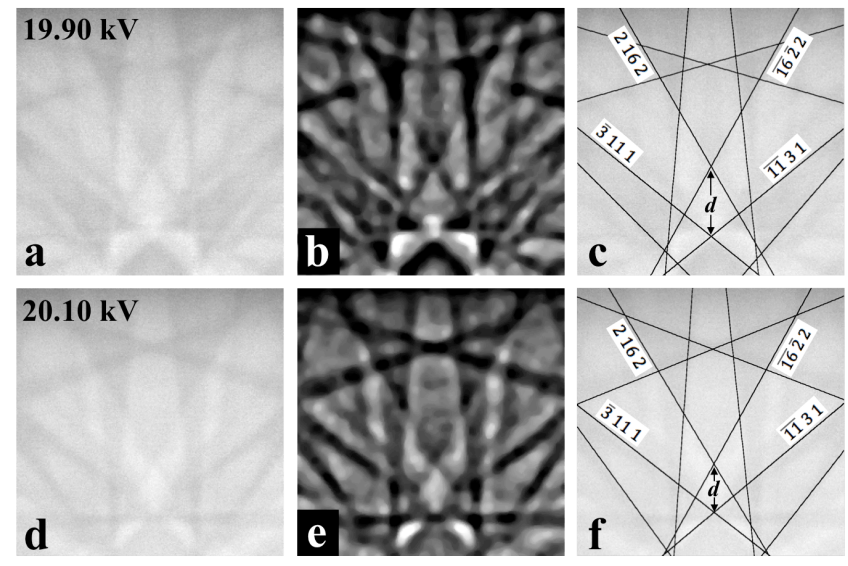

Figure 3. (a) $19.90 \mathrm{kV}$ ROI before and (b) after image processing. (c) Extracted HOLZ lines superimposed on the unprocessed ROI, depicting a distance $d$ between a selected pair of HOLZ line intersections. (d) $20.10 \mathrm{kV}$ ROI before and (e) after image processing. (f) Extracted HOLZ lines showing a measurable decrease in $d$.

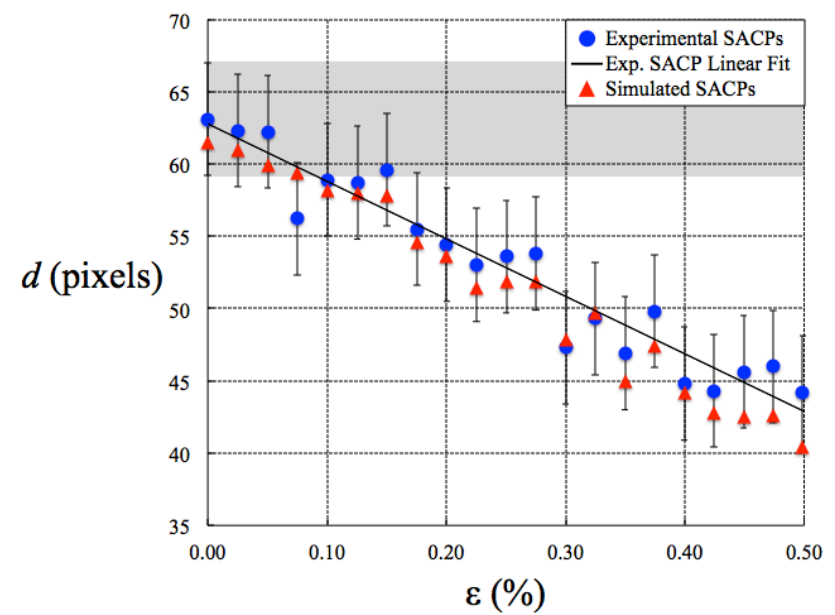

Figure 4. Plot showing relationship between the measured distance $d$ and the simulated hydrostatic strain. The shaded region is the measured $d$ of the "strain-free" (19.90 kV) experimental SACP and associated uncertainty. 